Randomised controlled trial

\section{Cannabinoids fail to show evidence of slowing down the progression of multiple sclerosis}

10.1136/ebmed-2015-110198

\section{Gareth Pryce, David Baker}

Department of Neuroscience, Queen Mary University of London, London, UK

Correspondence to: Dr Gareth Pryce, Centre for Neuroscience, Blizard Institute, Barts and The London School of Medicine and Dentistry, QMUL, 4 Newark Street, London E1 2AT, UK; g.pryce@qmul.ac.uk

Commentary on: Ball S, Vickery J, Hobart J, et al. The Cannabinoid Use in Progressive Inflammatory brain disease (CUPID) trial: a randomised double-blind placebo-controlled parallel-group multicentre trial and economic evaluation of cannabinoids to slow progression in multiple sclerosis. Health Technol Asess 2015;19:1-187.

\section{Context}

Despite the increasing number of effective disease modifying therapies for multiple sclerosis (MS), there are no effective therapies for the progressive, neurodegenerative phase of the disease. There is now abundant experimental evidence that cannabinoids and the endocannabinoid system are protective factors in animal models of MS and also other neurodegenerative conditions. This randomised controlled trial (RCT) aimed to investigate the potential neuroprotective properties of the prototypical cannabinoid $\Delta$ 9-tetrahydrocannabinol (THC) as measured by the slowing of the progressive phase of MS, based on data from animal models of $\mathrm{MS}^{1}$ and also clinical findings from an earlier study by the same group investigating the efficacy of THC as a spasticity alleviating therapy in $\mathrm{MS}^{2}$

\section{Methods}

Four hundred and eighty-nine eligible patients were enrolled in this blinded multicentre RCT, 332 received active treatment (oral dronabinol/ THC at maximal dose of $28 \mathrm{mg} /$ day) and 166 received placebo (vegetable oil capsules). Primary clinical outcome measures were: time to confirmed Extended Disability Status Scale (EDSS) score progression (physician based) at 3, 6 then 6 monthly up to 36 months unless progression was seen at this time when a further assessment was scheduled at 42 months and change in Multiple Sclerosis Impact Scale-29 (MSIS-29) score (patient based). Randomisation was balanced according to the EDSS score, study site and disease type and allocation of the randomisation scheme was concealed.

\section{Findings}

The study failed to demonstrate slowing of progressive disease on primary (time to confirmed EDSS score progression and MSIS-29) or secondary outcome measures (clinical and MRI measurement of brain atrophy). A treatment effect on slowing of progression rate was detected in a subgroup of patients (114 in active group, 46 in placebo group) with initial EDSS scores 4-5.5.

\section{Commentary}

This study highlights the problems in designing trials to investigate potential neuroprotective therapies in neurodegenerative conditions such as MS. It is clear from the results of this study that the inclusion of people with MS with an EDSS of 6 and above is problematic as, stated also by the authors, the EDSS is not linear and patients tend to spend considerably more time at EDSS 6-6.5 whereas progression through grades 4-5.5 tends to be more rapid. Clearly patients with EDSS scores of 6 and above are unlikely to be sensitive to a treatment effect during the typical course of this type of study (3-4 years) and there was a lower than expected progression rate observed in this study which may have affected the ability to detect clinical change, which would require longer trials.

While it was clear that daily THC, at the doses tested, did not slow progression it is of immense interest that analysis of a subset of people with MS with an EDSS 4-5.5 demonstrated that THC significantly $(p \leq 0.01)$ did slow disease progression but numbers were insufficient for firm conclusions to be drawn. ${ }^{3}$

Individuals with lower levels of disability should be considered for study in future clinical trials for neuroprotective therapies in MS, and thus, this study has performed a valuable service in indicating better trial design for future studies. However, the likelihood of further funding being obtained to perform a further RCT investigating the neuroprotective properties of cannabinoids may be unlikely and so a promising potential therapy for progressive MS may be lost.

Furthermore, although the trial was not designed for symptom control, it failed to demonstrate any impact on spasticity and so did not provide replication of the result ${ }^{2}$ on which the study rationale was based.

\section{Implications for practice}

This study does not provide evidence for a change in current practice, but it does indicate that in some patients THC can act as a neuroprotective agent, which requires further study to confirm this finding. ${ }^{3}$

\section{Competing interests None declared.}

Provenance and peer review Commissioned; internally peer reviewed.
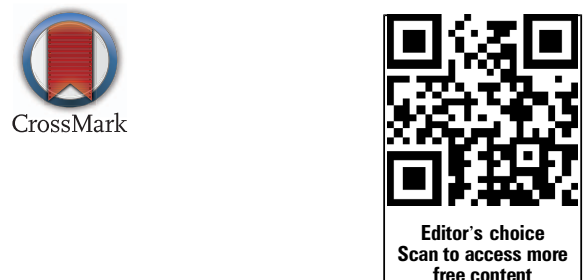

\section{References}

1. Pryce G, Ahmed Z, Hankey DJ, et al. Cannabinoids inhibit neurodegeneration in models of multiple sclerosis. Brain 2003;126:2191-202.

2. Zajicek JP, Sanders HP, Wright DE, et al. Cannabinoids in multiple sclerosis (CAMS) study: safety and efficacy data for 12 months follow up. J Neurol Neurosurg Psychiatry 2005;76:1664-9.

3. Pryce G, Riddall DR, Selwood DL, et al. Neuroprotection in experimental autoimmune encephalomyelitis and progressive multiple sclerosis by cannabis-based cannabinoids. J Neuroimmune Pharmacol 2014. 